\title{
Origin of Cosmic Rays: Modern status
}

\author{
Vladimir Ptuskin ${ }^{\mathrm{a}}$ \\ IZMIRAN, Troitsk, Moscow 108840, Russia
}

Abstract. Brief discussion on the origin of cosmic rays at energies from $10^{6}$ to $10^{20} \mathrm{eV}$.

\section{Introduction}

The directly measured cosmic-ray energy spectrum extends from about $1 \mathrm{MeV}$ to more than $10^{20} \mathrm{eV}$. The radio-astronomical, X-ray, gamma-ray observations and the first detection of very high energy astrophysical neutrinos shed light on the physical processes with cosmic rays. The majority of the observed cosmic rays are of Galactic origin and are accelerated in supernova remnants and pulsars. The Fermi Bubble is a prominent galactic source of energetic particles in the central part of the Galaxy. The origin of observed cosmic rays with energies above $\sim 10^{18} \mathrm{eV}$ is associated with extragalactic sources: Active Galactic Nuclei, progenitors of GammaRay Bursts, fast spinning newborn pulsars, large-scale structure formation shocks and some other objects.

\section{Last decade progress in cosmic ray research}

The last decade can be rightfully considered as a "golden age" of new cosmic-ray measurements. The list of remarkable experiments includes amongst others the Voyagers 1,2 traveling throughout the outer heliosphere and the interstellar medium; ACE, PAMELA and AMS spacecraft missions; long-duration balloon experiments ATIC, BESS, CREAM and TRACER; IceCube Neutrino Observatory at the South Pole; the ground based Extensive Air Shower (EAS) detectors KASCADEGrande, MILAGRO, ARGO-YBJ and Tunka; Auger and Telescope Array for studies of EAS produced by the highest-energy cosmic rays; the Fermi gamma-ray space observatory and the ground based HESS, MAGIC and VERITAS telescopes for gamma-ray observations of cosmic ray sources. The references to the experimental results of cosmic ray research can be found in [1].

The detection of astrophysical neutrinos with $\mathrm{PeV}$ and sub-PeV energies in the IceCube experiment [2] has opened a new way to study the sources of very high energy cosmic rays. The observed neutrino events are scattered over the sky without evident correlation with any Galactic or extragalactic classes of astronomical objects. The discussion about potential sources of very high energy neutrinos can be found in [3]. The measured neutrino flux is close to the Waxman-Bahcall upper bound [4] calculated

a e-mail: vptuskin@izmiran.ru under the assumptions that neutrinos are produced by ultrahigh energy cosmic rays in a process of their exit from the sources. However, the observed neutrino energy spectrum has a cutoff at $\sim 2 \mathrm{Pev}$ that corresponds to the maximum energy of primary protons $\sim 10^{17} \mathrm{eV}$, much smaller than the maximum energy of cosmic rays observed at the Earth. It was suggested [5] that extragalactic Type IIn supernova remnants are sources of observed diffuse high energy neutrinos. Based on the well developed theory of cosmic ray shock acceleration in supernova remnants it was shown that the large kinetic energy of explosion and very high gas density in the acceleration region (in the wind of a progenitor star with large mass loss rate) typical for Type IIn supernovae lead to the required energy of accelerated protons and efficiency of neutrino production in $\mathrm{pp}$ interactions.

Cosmic rays of the highest energies continue to hold the great interest of physicists. The HiRes, Auger and Telescope Array installations experimentally established the suppression of cosmic ray flux starting at energy about $5 \times 10^{19} \mathrm{eV}$ as expected due to the GreisenZatsepin-Kuzmin effect, the energy loss of the highest energy cosmic rays interacting with the extragalactic microwave and optic background radiation (see review [6]). The development of detailed models of acceleration and propagation of the highest energy cosmic rays is hampered by the uncertainty of the mass composition. The interpretation of HiRes and Telescope Array data favours the proton composition at energies $10^{18}$ to $5 \times$ $10^{19} \mathrm{eV}$, whereas the Auger data indicate that cosmic ray composition is becoming heavier for energies above $2 \times$ $10^{18} \mathrm{eV}$. The interpretation of recent results of the Auger experiment [7] favors a very hard source spectrum $\sim E^{-1}$ with mixed composition. The maximum particle magnetic rigidity at the sources $R_{\max } \approx 5 \times 10^{18} \mathrm{~V}$ corresponds to the maximum energy $\approx 1.3 \times 10^{20} \mathrm{eV}$ for Iron nuclei. This has the very important consequence that the shape of the all particle spectrum is likely due to the concurrence of two effects: maximum energy reached at the sources and energy losses during propagation.

The Voyger 1 and 2 spacecrafts provide direct measurements of cosmic ray spectrum and composition on opposite side of the energy spectrum. Launched in 1977 and expected to operate till 2020-2025, Voyager 1 is 138 a.u. from the Sun at present and Voyager 2 is at 114 a.u.. Voyager 1 is in "interstellar space" and Voyager 2 is currently in the "heliosheath" - the outermost layer

(c) The Authors, published by EDP Sciences. This is an Open Access article distributed under the terms of the Creative Commons Attribution License 4.0 (http://creativecommons.org/licenses/by/4.0/). 
of the heliosphere where the solar wind is slowed by the pressure of interstellar gas. The Voyager 1 spacecraft is measuring the cosmic ray intensity not affected by the solar wind modulation. This experiment extends our knowledge of the local interstellar cosmic ray spectrum and composition down to $\mathrm{MeV}$ energies [8]. The spectra of protons, nuclei and electrons look as a natural continuation of spectra at energies above a few hundred $\mathrm{MeV}$ and no additional low energy component was revealed. The intensity of the so called Anomalous Cosmic Rays (ACR) is diminished to zero and it confirms that these particles are accelerated somewhere in the inner part of the heliosheath (probably, at the hot spots of the solar wind termination shock).

The remarkable result of the PAMELA experiment was the detection of an unexpectedly large flux of positrons with energies above $10 \mathrm{GeV}$ [9]. It was confirmed with higher statistics and extended to higher energies by the AMS-02 experiment [10,11]. The measured flux of positrons is larger than the calculated flux of secondary positrons produced in cosmic ray interactions with interstellar gas atoms. The AMS-02 positron measurements are extended now up to $600 \mathrm{GeV}$ and show the rapid turn down of the spectrum above $250 \mathrm{GeV}$. The high content of positrons can be explained by the contribution of high-energy electron-positron pairs generated by pulsars $[12,13]$ or by the acceleration of positrons in the reverse shocks moving through the radioactive ejecta in supernova remnants [14]. The conclusive elucidation of the nature of positron flux in cosmic rays is very important since an alternative explanation suggests that these positrons are the products of dark matter decay.

Recent high-accuracy ATIC-2, CREAM and PAMELA measurements revealed deviations of the cosmic ray spectrum from a plain power law at energies from 10 to $10^{5} \mathrm{GeV} / \mathrm{n}[15,16]$. These deviations include the spectral hardening in proton, helium and nuclear spectra at magnetic rigidity $\sim 240 \mathrm{GV}$ and an increase of the $\mathrm{He} / \mathrm{p}$ ratio with energy. One of the explanations of these features implies cosmic ray acceleration by reverse shocks in supernova remnant [17]. New experimental results require the improvement of existing models of cosmic ray acceleration and propagation in the Galaxy although the basic model with the cosmic ray acceleration in supernova remnants (including pulsars) followed by the propagation in galactic magnetic fields with a slow leakage from the Galaxy remains immutable.

The fine structure of the all particle cosmic ray spectrum at $10^{5} \mathrm{GeV}$ to $10^{9} \mathrm{GeV}$ and the data on elemental composition were clarified in the KASCADE-Grande and Tunka experiments $[18,19]$. This structure corresponds to the superposition of fluxes of different nuclei with similar spectra on magnetic rigidity and the breaks (knees) at $3 \times 10^{6} \mathrm{ZGV}$ ( $Z$ is the particle charge). It results in the all-particle spectrum with a proton-helium knee at $3 \times 10^{6} \mathrm{GeV}$ and the steepening of a power law energy spectrum after this energy. The second knee at energy just below $10^{17} \mathrm{eV}$ where composition is dominated by heavy (iron) nuclei was found. The hard light component emerges at about $10^{17} \mathrm{eV}$. This component may be of Galactic origin or reflect the transition to extragalactic cosmic rays.

Efforts of theorists in the last few decades were mainly focused on the problem of particle acceleration by shocks propagating in the turbulent interstellar plasma $[20,21]$. The modeling of nonlinear shock acceleration in the different types of supernova remnants demonstrated that the calculated energy spectrum of cosmic rays after propagation through the Galaxy is in good agreement with the measured spectrum up to approximately $3 \times 10^{18} \mathrm{eV}[22]$.

\section{Dark matter problem and cosmic rays}

An important aspect of cosmic ray research is associated with a fundamental problem of physics the clarification of the nature of dark matter in the Universe. It is established that the energy budget of the Universe consists of only $4 \%$ of baryonic matter, $73 \%$ is made of the dark energy and the $23 \%$ of dark matter. Dark matter particles are not described by the Standard Model of elementary particle physics. The promising candidates for dark matter are the WIMPs, weakly interacting massive particles with a mass in the range between tens of $\mathrm{GeV}$ and $\mathrm{TeV}$. A direct search for weak interactions of these particles with large mass detectors is conducted in underground laboratories. The indirect search in space and at the top of the atmosphere is based on the assumption that WIMPs can annihilate each other and produce standard elementary particles as the final state. Using gamma-astronomical methods, one can search for specific signals in continuum and line radiation from galaxies, satellite galaxies, galaxy clusters, Galactic Center and other regions (clumps) where dark matter may be accumulated. The gamma-ray space telescope Fermi is used for this purpose [23]. The cosmic ray antiparticles, positrons and antiprotons, are convenient to use for the dark matter search. They are produced only in small amounts via interactions of CRs with interstellar matter and it is easier to disentangle dark matter signal from the astrophysical background (see e.g. discussion in [24]).

As was said above, the anomalously high flux of positrons in cosmic rays can, in principle, be explained by the contribution from pulsars or the production of positrons via decays of dark matter.

Antiprotons in cosmic rays are produced as secondary particles at a level $\sim 10^{-4}$ relative to protons. Accurate antiproton measurements done at $70 \mathrm{MeV}$ to $200 \mathrm{GeV}$ by the PAMELA instrument [25] and up to $400 \mathrm{GeV}$ by AMS02 [11] did not reveal any unambiguous evidence of excess above the predicted secondary antiproton flux although some preference for a flatter component probably starts to emerge above $100 \mathrm{GeV}$ [26,27].

No firm evidence of dark matter signal was found so far, but the investigations of cosmic rays play an important role in constraining dark-matter models for understanding the nature of dark matter (see [28,29]).

The present work was partly supported by RFBR grant 16-02-00255.

\section{References}

[1] K.A. Olive et al. (Particle Data Group), Chinese Phys. C 38, 090001 (2014)

[2] IceCube Collaboration, Science 342, 1242856 (2013)

[3] T. Gaisser, F. Halzen, Annu. Rev. Nucl. Part. Sci. 64, 101 (2014)

[4] E. Waxman, J. Bahcall, Phys. Rev. 59, 023002 (1999) 
[5] V.N. Zirakashvili, V.S. Ptuskin, Astropart. Physics 78, 28 (2016)

[6] K. Kotera, A. V. Olinto, Annu. Rev. Astron. Astrophys. 49, 119 (2011)

[7] A. Aab et al., arXiv: 16107155 (2016)

[8] E. Stone et al., Science 341, 150 (2013)

[9] O. Adriani et al., Nature 458, 607 (2009)

[10] M. Aguilar et al., Phys. Rev. Lett. 113, 121102 (2014)

[11] S. Ting, AMS days at CERN https://indico.cern.ch/ event/381134 (2015)

[12] A. Harding, R. Ramaty, Proc. 20th Intern. Cosmic Ray Conf. Moscow 2, 92 (1987)

[13] M. Di Mauro et al., JCAP 4, 006 (2014)

[14] V.N. Zirakashvili, F.A. Aharonian, Phys. Rev. D 84, 083010 (2011)

[15] O. Adriani et al., Science 332, 69 (2011)

[16] H.S. Ahn et al., ApJ Lett. 714, L89 (2010)

[17] V.S. Ptuskin, V.N. Zirakashvili, E.S. Seo, ApJ 763, 47 (2013)
[18] A. Haungs et al., Proc. 33rd Intern. Cosmic Ray Conf. Rio de Janeiro, arXiv:1308.1485v1 (2013)

[19] V.V. Prosin et al., Nucl. Instrum. Methods in Physics Research A 756, 94 (2014)

[20] A.R. Bell, Astropart. Physics 43, 56 (2013)

[21] D. Caprioli, Nucl. Phys. B (Proc. Suppl. 256, 48 (2014)

[22] V.S. Ptuskin, V.N. Zirakashvili, E.S. Seo, ApJ 718, $31(2010)$

[23] W. B. Atwood et al., ApJ 697, 1071 (2009)

[24] P. Picozza, R. Sparvoli, NIMPA 729, 45 (2013)

[25] O. Adriani et al., Phys.Rev. Lett. 111, 1102 (2013)

[26] G. Giesen et al., JCAP 1509, 023 (2015)

[27] R. Kappl, A. Reinert, M.W. Winkler, JCAP 10, 034 (2015)

[28] G. Beck, S. Colafrancesco, arXiv150801386 (2015)

[29] K. Hamaguchi, T. Mori, K. Nakayama, Phys. Lett. B 747, 523 (2015) 